In conclusion, $\mathrm{FEV}_{1} / \mathrm{FIV}_{1}$ index has a good correlation with ALSFRS-R $\left(\mathrm{n}=20, \mathrm{r}=-0.71, \mathrm{p}<0.001, \mathrm{FEV}_{1} / \mathrm{FIV}_{1}=1.630-\right.$ $[0.018 *$ ALSFRS-R] \pm 0.165$)$.

\section{P212 PARASTERNAL INTERCOSTAL ELECTROMYOGRAPHY TO ASSESS NEURAL RESPIRATORY DRIVE IN HEALTHY ADULT SUBJECTS}

${ }^{1} \mathrm{~V}$ MacBean, ${ }^{1} \mathrm{C}$ Hughes, ${ }^{1} \mathrm{G}$ Nicol, ${ }^{2} \mathrm{CC}$ Reilly, ${ }^{1} \mathrm{GF}$ Rafferty. ${ }^{1}$ King's College London, London, UK; ${ }^{2}$ King's College Hospital NHS Foundation Trust, London, UK

\subsection{6/thoraxjnl-2014-206260.341}

Neural respiratory drive (NRD), measured using the parasternal intercostal muscle electromyogram (EMGpara), relates to lung disease severity as quantified by conventional methods in a range of diseases. Reference data from healthy populations are required for the technique to be used as an independent measure of lung disease severity. EMGpara has previously been expressed as a percentage of that obtained during a maximal inspiratory effort (EMGpara\%max), restricting the use of the technique to subjects able to reliably perform such manoeuvres. The aim of this study was to investigate variability of both raw EMGpara (rEMGpara) and EMGpara\%max in healthy adults.

EMGpara was measured during tidal breathing in 43 healthy adult non-smokers (25 females, median (range) age 32 (19-79) years, mean (SD) BMI $23.4(3.5) \mathrm{kg} / \mathrm{m}^{2}$ ), using surface electrodes positioned bilaterally over the second interchondral space. Measurements were made with and without a mouthpiece/pneumotachograph in situ in 20 participants. Repeated measures were obtained within the same testing session in 27 subjects, and at least seven days later in 13 individuals. Spirometry, height, weight, BMI, fat free mass (FFM) via bioelectrical impedance and measures of regional fat distribution (waist/hip ratio and neck circumference) were also recorded.

Mean (SD) EMGpara\%max and rEMGpara were 5.88 (3.63)\% and $5.06(2.26) \mu \mathrm{V}$ respectively. Significant relationships were observed between anthropometric measures and rEMGpara and EMGpara\%max (Table 1). rEMGpara and EMGpara\%max were unrelated to spirometry variables. Median (range) rEMGpara and EMGpara\%max increased significantly with the pneumotachograph in place $(4.86(2.11-8.19) \mu \mathrm{V}$ versus 5.62 $(2.47-10.98) \mu \mathrm{V}$ and $4.77(1.68-17.00) \%$ versus 6.78 (2.35$20.94) \%$, both $\mathrm{p}<0.0001)$.

Analysis of variance by subject was used to assess within-subject variability. Measurement error was higher for EMGpara\%

\begin{tabular}{|c|c|c|}
\hline & $\begin{array}{l}\text { Correlation with raw } \\
\text { EMGpara }(r(p))\end{array}$ & $\begin{array}{l}\text { Correlation with } \\
\text { EMGpara\%max (r (p) }\end{array}$ \\
\hline & -0.38 & -0.60 \\
\hline \multirow[t]{2}{*}{ Height } & $(0.01)$ & $(<0.0001)$ \\
\hline & -0.47 & -0.57 \\
\hline \multirow[t]{2}{*}{ Weight } & $(0.001)$ & $(<0.0001)$ \\
\hline & -0.46 & -0.35 \\
\hline \multirow[t]{2}{*}{ BMI } & $(0.002)$ & $(0.02)$ \\
\hline & -0.12 & -0.28 \\
\hline \multirow[t]{2}{*}{ FFM } & (ns) & (ns) \\
\hline & -0.43 & -0.68 \\
\hline \multirow[t]{2}{*}{ Neck circumference } & $(0.004)$ & $(<0.0001)$ \\
\hline & -0.23 & -0.48 \\
\hline Waist/hip ratio & (ns) & $(0.001)$ \\
\hline
\end{tabular}

max than rEMGpara (upper 95\% confidence limit of difference between repeat measures of EMGpara\%max 3.14\%, versus 2.35 $\mu \mathrm{V}$ for rEMGpara; within-subject coefficient of variation EMGpara\%max 30.8\% versus rEMGpara 24.5\%).

rEMGpara appears to be a reproducible marker of NRD. Both rEMGpara and EMGpara\%max are influenced by subjects' anthropometry. Further investigation is required to determine whether these influences are technical or physiological and must be considered when the technique is applied clinically or for research, or when developing reference values.

\section{P213 THE IMPACT OF SLEEP DISORDERED BREATHING ON PERIPHERAL MUSCLE}

${ }^{1} \mathrm{~S}$ Mandal, ${ }^{2} \mathrm{~T}$ Dhir, ${ }^{1} \mathrm{~A}$ Vaughan-France, ${ }^{1} \mathrm{ES}$ Suh, ${ }^{1} \mathrm{~N}$ Hart. ${ }^{1}$ Lane Fox Respiratory Unit, Guy's and St Thomas' Hospital, London, UK; ' King's College London, London, UK

\subsection{6/thoraxjn-2014-206260.342}

Introduction Chronic obstructive pulmonary disease is characterised by peripheral muscle wasting with consequent reduction in muscle strength and function. In this cohort of patients a reduction in muscle strength correlates with morbidity and mortality. Less well known are the characteristics of muscle in patients with sleep disordered breathing (SDB), a disease state that can also be dominated by inflammation, breathlessness and hypoxia. We sought to examine the impact of sleep disordered breathing on peripheral muscle size and strength.

Method 51 subjects were recruited: 15 healthy controls (HC) with a normal body mass index (BMI, $<25 \mathrm{~kg} / \mathrm{m}^{2}$ ), 16 overweight and obese individuals with no SDB controls (SO), and 20 obese subjects with obstructive sleep apnoea (OSA). Subjects underwent measurements of Rectus Femoris Cross Sectional Area $\left(\mathrm{RF}_{\mathrm{CSA}}\right)$ and quadriceps maximal voluntary contraction

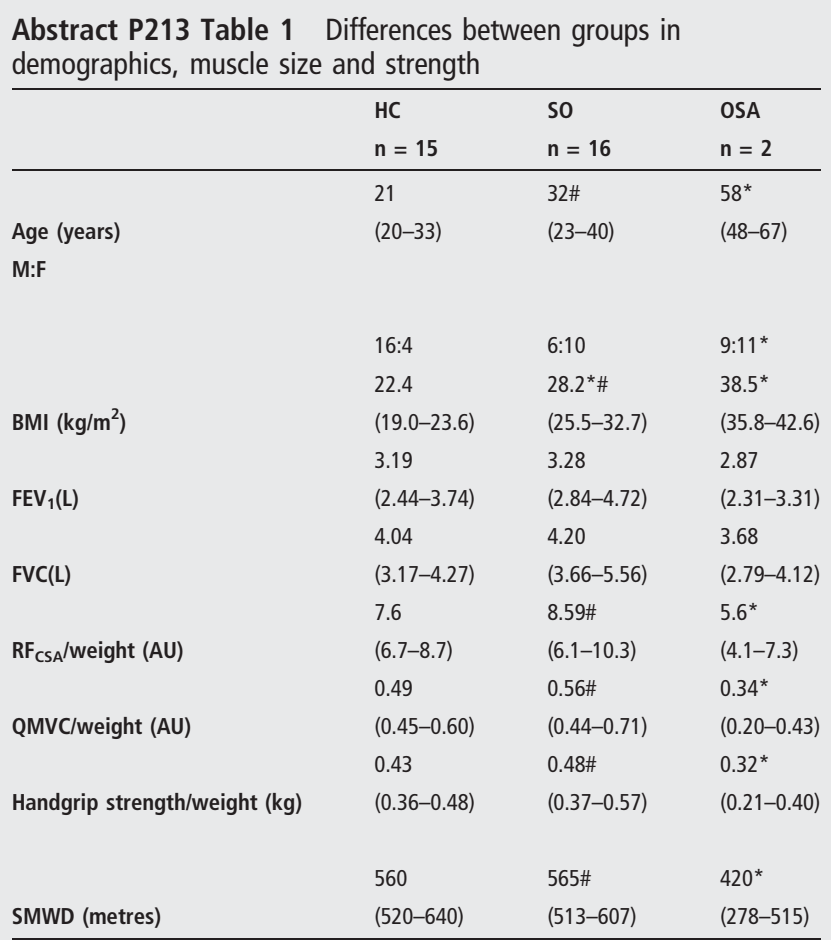

*significantly different from HC $(p<0.05)$; \#significantly different from OSA $(p<0.05)$ Abbreviations: $\mathrm{HC}=$ Healthy Controls, $\mathrm{SO}=$ Simple Obesity/Overweight, $\mathrm{OSA}=0 \mathrm{bstructive}$ Sleep Apnoea, BMI-Body Mass Index, $\mathrm{RF}_{\mathrm{CSA}}=$ Rectus Femoris Cross Sectiona Area, QMVC=Quadriceps Maximal Voluntary Contraction, SMWD=Six Minute Walking Distance 